\title{
CTA site characterization: a contribution on Sky Background Brightness
}

\author{
Gonzalo de la Vega ${ }^{1}$, Beatriz García ${ }^{1}$, Javier Maya ${ }^{1}$, Alexis Mancilla ${ }^{1}$ \\ and Ezequiel Rosemblat ${ }^{2}$ \\ ${ }^{1}$ ITeDA Mendoza, CNEA,CONICET, UNSAM \\ Azopardo 3135501 Godoy Cruz, Mendoza, Argentina \\ email: beatriz.garcia@iteda.cnea.gov.ar \\ ${ }^{2}$ Universidad Mendoza, Mendoza, Argentina
}

\begin{abstract}
The Cherenkov Telescope Array (CTA) is an international project for which more than 1000 scientists from 27 countries work together to install two observatories, one in the southern hemisphere and the other in the north, to study gamma-ray radiation. Argentina is a candidate site for one of the observatories, and to characterize proposed sites, one must study the sky background brightness. This critical point must be studied with care. Nearest cities can be a decisive factor in determining where to locate an observatory in our times.
\end{abstract}

Keywords. Sky Background Brightness, Sky Background Brightness: Observatories site selection

Background sky emission is studied for site characterization for the installation of astronomical observatories. Atmospheric emission has two main sources, one from Earth (air glow, activity by auroras and thermal conditions) and one from outer space (contributions from the moon, stars or galaxies, zodiacal light, and diffuse background from our galaxy). The first depends on different variables (seasons, solar activity, geographical location), but the second is connected to the telescope pointing position. The extraterrestrial sources are due to unresolved stars and galaxies and, the most important, sunlight scattered by interplanetary dust. (Patat (2006), Patat (2008)).

In recent years, many observatories and groups devoted to site selection to install telescopes or astronomical instrumentation have used commercial devices such as the Sky Quality Meter (SQM) manufactured by Unihedron (http : //www.uniheadron.com). This equipment is a very simple photometer, easy to install, inexpensive and can be used in different sites at the same time.

In 2012, we installed two SQM-LR devices in Argentina at the CTA candidate sites (El Leoncito in San Juan, and San Antonio de los Cobres in Salta). The instruments were calibrated at the laboratory and cross-calibrated with adapted SQM-L instruments installed at the same sites since March 2011. The results from the instruments are comparable, but cross calibration shows that a correction (of about $0.4 \mathrm{mag} / \mathrm{arcsec}^{2}$ ) must be applied to the data. This implies that if different devices are used (i.e. SQM-L and SQ-LR) and if precise results are needed, cross calibration between them is always necessary.

We determine that the effect of a single star of low magnitude is negligible in SQM measurements, but 15 stars of magnitude 4 would increase the background by 0.23 $\mathrm{mag} / \operatorname{arcsec}^{2}$. This situation is worst during the transit of the Milky Way across the instrument's field of view; the bulge of the Galaxy contributes about one magnitude.

\section{References}

Patat, F., ESO Internal Report, 2006. (http://www.eso.org/ fpatat/science/skybright/)

Patat, F., $A$ \& $A, 481,575-591,2008$ 